Conversely, ROS generation may help T. cruzi thrive in an environment of high oxidative stress by allowing superoxidemediated iron release from ferritin, and/ or peroxide-mediated iron extraction from heme and/or iron-sulfur cluster proteins (ref. 19 and Figure 1). Importantly, in this scenario the stimulation of T. cruzi growth by oxidative stress would not be restricted to macrophages, since a persistent oxidative environment can be generated by the parasites in other cell types, such as cardiomyocytes (7). It will be very interesting to determine whether Leishmania parasites, which are closely related to $T$. cruzi but replicate in the endocytic compartment of macrophages, also fare better under oxidative stress. Such studies may provide an answer to the fascinating cell biological question of whether residence in the cytosol or within a membrane-bound compartment has a differential impact on the access of pathogens to iron, on the specific iron pools utilized, and on the consequences of oxidative stress.

\section{Acknowledgments}

Work in the author's laboratory is suppoted by NIH grants R01AI067979, R01GM064625, and R37AI34867. The author thanks Bidyottam Mittra and Iqbal Hamza for critical reading of the article, and all members of her laboratory for stimulating discussions.

Address correspondence to: Norma W. Andrews, Department of Cell Biology and Molecular Genetics, 2134 Bioscience Research Building, University of Maryland, College Park, Maryland 20742-5815, USA. Phone: 301.405.8418; Fax: 301.314.9489; E-mail: andrewsn@umd.edu.

1. Paiva $\mathrm{CN}$, et al. Oxidative stress fuels Trypanosoma cruzi infection in mice. J Clin Invest. 2012; 122(7):2531-2542

2. Poss KD, Tonegawa S. Reduced stress defense in heme oxygenase 1-deficient cells. Proc Natl Acad Sci US A. 1997;94(20):10925-10930.

3. Muller S, Liebau E, Walter RD, Krauth-Siegel RL. Thiol-based redox metabolism of protozoan parasites. Trends Parasitol. 2003;19(7):320-328.

4. Andrade LO, Andrews NW. The Trypanosoma cruzi-host-cell interplay: location, invasion, retention. Nat Rev Microbiol. 2005;3(10):819-823.

5. Vago AR, et al. Kinetoplast DNA signatures of Trypanosoma cruzi strains obtained directly from infected tissues. Am J Pathol. 1996;149(6):2153-2159.

6. Tarleton RL, Zhang L, Downs MO. "Autoimmune rejection" of neonatal heart transplants in experimental Chagas disease is a parasite-specific response to infected host tissue. Proc Natl Acad Sci US A. 1997;94(8):3932-3937.

7. Gupta S, Dhiman M, Wen JJ, Garg NJ. ROS signalling of inflammatory cytokines during Trypano- soma cruzi infection. Adv Parasitol. 2011;76:153-170.

8. Kaplan J. Mechanisms of cellular iron acquisition: another iron in the fire. Cell. 2002;111(5):603-606.

9. Marro S, et al. Heme controls ferroportin1 (FPN1) transcription involving Bach1, Nrf2 and a MARE/ ARE sequence motif at position -7007 of the FPN1 promoter. Haematologica. 2010;95(8):1261-1268.

10. Hintze KJ, Theil EC. DNA and mRNA elements with complementary responses to hemin, antioxidant inducers, and iron control ferritin-L expression. Proc Natl Acad Sci U S A. 2005;102(42):15048-15052.

11. Taylor MC, Kelly JM. Iron metabolism in trypanosomatids, and its crucial role in infection. Parasitology. 2010;137(6):899-917.

12. Lima MF, Villalta F. Trypanosoma cruzi receptors for human transferrin and their role. Mol Biochem Parasitol. 1990;38(2):245-252.

13. Wilson ME, Britigan BE. Iron acquisition by parasitic protozoa. Parasitology Today. 1998;14(9):348-353.

14. Borges VM, Vannier-Santos MA, de Souza W. Subverted transferrin trafficking in Leishmania-infected macrophages. Parasitol Res. 1998;84(10):811-822.

15. Flannery AR, Huynh C, Mittra B, Mortara RA, Andrews NW. LFR1 ferric iron reductase of Leishmania amazonensis is essential for the generation of infective parasite forms. J Biol Chem. 2011; 286(26):23266-23279.

16. Huynh C, Sacks DL, Andrews NW. A Leishmania amazonensis ZIP family iron transporter is essential for parasite replication within macrophage phagolysosomes. J Exp Med. 2006;203(10):2363-2375.

17. Blackwell JM, et al. SLC11A1 (formerly NRAMP1) and disease resistance. Cell Microbiol. 2001;3(12):773-784.

18. Marquis JF, Gros P. Intracellular Leishmania: your iron or mine? Trends Microbiol. 2007;15(3):93-95.

19. Kakhlon O, Cabantchik ZI. The labile iron pool: characterization, measurement, and participation in cellular processes(1). Free Radic Biol Med. 2002;33(8):1037-1046.

\title{
Fibroblast growth factor 23: friend or foe in uremia?
}

\author{
Orson W. Moe
}

Department of Internal Medicine, Department of Physiology, and Charles and Jane Pak Center of Mineral Metabolism, University of Texas Southwestern Medical Center, Dallas, Texas, USA.

\begin{abstract}
Uremia is a complex metabolic state marked by derangement of many signaling molecules and metabolic intermediates; of these, the massively increased levels of FGF23 are among the most striking. It has remained unclear whether FGF23 is directly implicated in the pathogenesis of chronic kidney disease (CKD) and its complications, a consequence of other dysregulated pathways, or perhaps an adaptive - and thus desirable - response. In this issue of the JCI, Shalhoub et al. describe the chronic effects of antibody-mediated FGF23 neutralization in a CKD mouse model, shedding new light on this complicated story and moving us one step closer to understanding the role of FGF23 in CKD.
\end{abstract}

Chronic kidney disease (CKD) is a global health problem that has a grave impact

Conflict of interest: The author has declared that no conflict of interest exists.

Citation for this article: J Clin Invest. 2012; 122(7):2354-2356. doi:10.1172/JCI64184. on morbidity and mortality rates. An extensive treatise published by the National Kidney Foundation in 1998 pointed out the sobering reality that a dialysis patient in his or her twenties has the same risk of dying as an 80-year-old person not on dialysis (1). Rather regret- tably, the situation has not improved dramatically in the interceding years despite numerous efforts to understand the pathophysiology and improve treatment of CKD. Renal replacement therapy and organ transplant are undoubtedly life-saving implementations, but these options are reserved for end-stage renal failure and harbor their own limitations; dialysis is restricted to clearance of a limited group of molecules and therefore not a true "renal replacement," and organ transplant is complicated by the requirement for universal suppression of the immune system. How can one devise additional strategies to significantly improve both life span and quality of life of patients with CKD? 


\section{The readouts of renal dysfunction}

Uremia is a highly complex metabolic state. Since the kidney is the principal organ that preserves the constancy of the milieu intérieur, eloquently articulated by Claude Bernard (2), the impact of its dysfunction is widespread and immense. When renal function is compromised, exogenous uremic toxins accumulate and endogenous substances slip to levels incompatible with health. Secondary derangements further compound the state of affairs, cumulating in a downward unrelenting spiral to ultimate metabolic cataclysm. To realistically treat the uremic state, one must devise therapeutic interventions specifically targeted to prevent, arrest, and reverse each of the individual abnormalities of pathogenic significance.

While apparently straightforward in principle, this task is in fact extremely difficult. The derangement of a measurable parameter could merely be a biomarker (e.g., hypercreatinemia), could reflect a pathogenic factor (e.g., hyperphosphatemia, or both, and it is not always easy to distinguish between the two. A mild elevation of urea may be no more than a marker but higher elevations may in fact be deleterious (3). The current count of "uremic toxins" is in the three-digit range (4). Which should clinicians target and how should we prioritize? Does one always aim to bring a parameter back to its normal range as defined in the nonuremic state? This is certainly not true for hematocrit and parathyroid hormone (PTH) (5-7), where a "new normal" for the uremic state is yet to be identified. Even for a simple marker such as urea, the effect on clinical outcome follows a "J curve," so "thelower-the-better" approach is not universally valid. An important factor to consider in this context is FGF23.

\section{Troublemaker, bystander, or wrongly accused?}

Since the cloning of FGF23 based on mutations in uncommon diseases $(8,9)$, this peptide has earned a reputation as the new "bad guy in town" in the CKD world. The current view of FGF23 is that it is one of the most important phosphate-regulatory hormones that acts on multiple organs to maintain homeostasis (10). It is quite unusual to witness an endocrine disorder in which the level of the deranged hormone reaches three orders of magnitude higher than the normal state. However, one encounters one epidemiologic study after another that links such high FGF23 levels to poor clinical out- comes (reviewed in ref. 11). Notwithstanding the fact that negative studies are less likely to be published, we are still left with an unprecedented and overwhelming collection of clinical literature linking high FGF23 levels to adverse outcomes, leaving most practitioners to conclude that FGF23 must contribute negatively to the uremic state. The power of epidemiology is of course the sample size, with data secured "in the field," but it suffers from the perpetual limitation of mere association without the power to conclude on causality. When confronted with this body of data, one ponders whether high FGF23 is a mere marker for some underlying sinister menace or a bona fide uremic toxin yet to be caught with the smoking gun. A third paradigm - perhaps considered by some as borderline preposterous - is that the sickest CKD patients may require the highest FGF23 levels, suggesting that FGF23 could be a defensive hormone. Could FGF23 be a "good guy"? Clearly, the current body of evidence cannot proffer definitive proof, but is sufficient to spawn efforts to devise an approach to neutralize the colossally high FGF23 levels in CKD and simply see what happens.

\section{Neutralizing FGF23}

While receptor and receptor signaling antagonists are logical candidates for this approach (12-14), two groups proceeded to attempt FGF23 neutralization with therapeutic antibodies (15-17). Both groups reported that, acutely, anti-FGF23 monoclonal antibodies suppress PTH and raise vitamin $\mathrm{D}$ levels, likely via indirect and direct effects, respectively. These effects are nice confirmations of what we know about FGF23 biology and indeed are favorable from the viewpoint of potential therapeutics in CKD. In this issue of the JCI, one of those groups describes the chronic effects of the therapeutic antibody on the pathology in CKD in animal models (17). In this article, Shalhoub and coworkers showed several salient features of the administration of an anti-FGF23 monoclonal antibody of nanomolar affinity in rodents with an ablation model of CKD. The monoclonal antibody did not change the course of progression of CKD nor did it affect various left ventricular cardiac parameters. These findings are at odds with the proposed models of phosphotoxicity contributing to CKD progression (18) or direct "FGF23 cardiomyopathy" (12), which further illustrates the complexity of these paradigms. The improvement in static and dynamic bone histomorphometric data was impressive and may be related to the improved mineral metabolism (15). Despite the favorable changes in PTH and vitamin D levels, there was worsening hyperphosphatemia with antibody therapy with no change in phosphate excretion rate and very minor changes in fractional excretion of phosphate. Since data on external phosphate balance were not presented, one can only conjecture that the antibody may be promoting phosphate retention and phosphotoxicity.

\section{Friend, foe, or both?}

The most striking finding of Shalhoub et al. was the significant worsening of animal survival and aortic calcification and the accompanying logical - but presumptive - notion that the mice succumbed to cardiovascular deaths (17). These data may be disappointing to those anticipating emergence of a magic bullet for CKD, but the reality is that in this rodent model, the therapeutic anti-FGF23 antibody in this dosage exerts biologic effects that are far from favorable in overall survival. While the data may be unequivocal, the interpretation remains open. Does this finding permit one to conclude that the FGF23 elevation is in fact adaptive rather than pathophysiologic? Implicit in this view is the notion that one should not interfere with a natural adaptation intended to counter the metabolic disturbances in CKD. In fact, subscribers to this paradigm may wish to provide some assistance to this adaptive action by administering recombinant FGF23 to improve phosphate balance; that is, if neutralizing FGF23 is bad, perhaps should one elevate it instead. No doubt, this dichotomous view of FGF23 as either good or evil is far too simplistic. Despite the side effects of high PTH levels in CKD, we have learned that we cannot simply eliminate PTH. Likewise, if FGF23 is still one of the key phosphaturic hormones, one may need discreet levels in CKD to maintain phosphate balance. Complete obliteration would indeed be unwise, and given the findings of Shalhoub and coworkers (17), perhaps increased FGF23 levels would actually be therapeutic.

Another attractive but yet unproven view is the "off-target effect" theory. If deficiency of the coreceptor protein Klotho in CKD (19) is impairing the ability of FGF23 to exert its legitimate actions, the remarkably high FGF23 levels could activate off-target effects and stir up multiple rogue pathways. Indeed, this was proposed by Faul and coworkers in describing how the activation of FGF receptor- 2 may contribute to cardio- 
myopathy in CKD (12). If there is truth to this model, one may not want to obliterate FGF23 but rather direct FGF23 back to its more physiologic actions, perhaps by restoring Klotho levels.

\section{Looking ahead}

In sum, one should not view the study of Shalhoub and coworkers as a nihilistic "show stopper" in designing FGF23-related therapeutics in CKD. Instead, it should alert the field of the need to abandon the overly simplistic FGF23 toxicity model. Efforts should be directed toward defining the roles of FGF23 in physiology and CKD. What is actually driving up the FGF23 levels in CKD? What are the optimal levels in various stages of CKD for its different roles? What are the ways to enhance endogenous on-target signaling as adaptive reactions and decrease the off-target effects? Instead of finding the singular magic bullet, perhaps derangements of mineral metabolism need to be attacked at multiple loci. FGF23 is likely both friend and foe in CKD, and much knowledge and wisdom needs to be secured before we can move toward therapeutic applications.

\section{Acknowledgments}

The author is supported by the NIH, the Simmons Family Foundation, the Charles and Jane Pak Foundation, and an investigator-initiated GRIP research grant from Genzyme Corporation.

Address correspondence to: Orson W. Moe, Departments of Internal Medicine and Physiology, University of Texas Southwestern Medical Center, 5323 Harry Hines Blvd., Dallas, Texas 75390, USA. Phone: 214.648.7993; Fax: 214.648.2071; E-mail: Orson.Moe@utsouthwestern.edu.

1. [No authors listed]. Strategies for influencing outcomes in pre-ESRD and ESRD patients: a special conference. 1998. Proceedings. Am J Kidney Dis. 1998;32(6 suppl 4):S1-S193.

2. Bernard C. Introduction a L'etude de la Medecine Experimentale. Paris, France: Éditions GarnierFlammarion; 1865.

3. D'Apolito M, et al. Urea-induced ROS generation causes insulin resistance in mice with chronic renal failure. JClin Invest. 2010;120(1):203-213.

4. European Uremic Toxin (EUTox) Work Group of the ESAO and ERA-EDTA. EUTox Web site. www.uremic-toxins.org. Accessed May 23, 2012

5. Singh AK. What is causing the mortality in treating the anemia of chronic kidney disease: erythropoietin dose or hemoglobin level? Curr Opin Nephrol Hypertens. 2010;19(5):420-424.

6. The National Kidney Foundation Kidney Disease Outcomes Quality Initiative (NKF KDOQI). National Kidney Foundation Web site. http:// www.kidney.org/professionals/KDOQI/guidelines_commentaries.cfm. Accessed May 23, 2012.

7. Clinical practice guidelines. Kidney Disease: Improving Global Outcomes (KDIGO) Web site. http://www.kdigo.org/clinical_practice_guidelines/index.php. Accessed May 23, 2012.
8. ADHR-Consortium. Autosomal dominant hypophosphataemic rickets is associated with mutations in FGF23. Nat Genet. 2000;26(3):345-348

9. Shimada $\mathrm{T}$, et al. Cloning and characterization of FGF23 as a causative factor of tumor-induced osteomalacia. Proc Natl Acad Sci U S A. 2001; 98(11):6500-6505.

10. Martin A, David V, Quarles LD. Regulation and function of the FGF23/klotho endocrine pathways. Physiol Rev. 2012;92(1):131-155.

11. Isakova T. Fibroblast growth factor 23 and adverse clinical outcomes in chronic kidney disease. Curr Opin Nephrol Hypertens. 2012;21(3):334-340.

12. Faul C, et al. FGF23 induces left ventricular hypertrophy. J Clin Invest. 2011;121(11):4393-4408.

13. Goetz R, et al. Isolated C-terminal tail of FGF23 alleviates hypophosphatemia by inhibiting FGF23FGFR-Klotho complex formation. Proc Natl Acad Sci US A. 2010;107(1):407-412

14. Razzaque MS. Therapeutic potential of klothoFGF23 fusion polypeptides: WO2009095372. Expert Opin Ther Pat. 2010;20(7):981-985.

15. Hasegawa $\mathrm{H}$, et al. Direct evidence for a causative role of FGF23 in the abnormal renal phosphate handling and vitamin D metabolism in rats with early-stage chronic kidney disease. Kidney Int. 2010;78(10):975-980

16. Yamazaki Y, et al. Anti-FGF23 neutralizing antibodies show the physiological role and structural features of FGF23. J Bone Miner Res. 2008; 23(9):1509-1518.

17. Shalhoub V, et al. FGF23 neutralization improves chronic kidney disease-associated hyperparathyroidism yet increases mortality. J Clin Invest. 2012; 122(7):2543-2553.

18. Zoccali C, et al. Phosphate may promote CKD progression and attenuate renoprotective effect of ACE inhibition. J Am Soc Nephrol. 2011;22(10):1923-1930.

19. Hu MC, et al. Klotho deficiency and vascular calcification in chronic kidney disease. J Am Soc Nephrol. 2011;22(1):124-136

\title{
Pharmacogenomics: mapping monogenic mutations to direct therapy
}

\author{
Palmer Taylor \\ Department of Pharmacology, Skaggs School of Pharmacy and Pharmaceutical Sciences, UCSD, La Jolla, California, USA.
}

\begin{abstract}
The molecular mapping of mutations that underlie congenital disorders of monogenic origin can result in both a broader understanding of the molecular basis of the disorder and novel therapeutic insights. Indeed, genotyping patients and then replicating the behavior of the mutant gene products in welldefined biochemical or electrophysiological systems will allow tailoring of therapy to be mutation- and protein sequence-dependent. In this issue of the JCI, Shen and colleagues describe such an approach that identified novel mutations in the $\alpha$ subunit of the nicotinic receptor linked to myasthenia gravis.
\end{abstract}

In this issue of the JCI, Shen and colleagues detail the discovery of novel mutations

Conflict of interest: The author holds stock in Merck, Pfizer, Sequenom, Qualcom, Lilly, and Abbott and receives research support from Targacept.

Citation for this article: J Clin Invest. 2012; 122(7):2356-2358. doi:10.1172/JCI64409. linked to myasthenia gravis (1), a disease characterized by skeletal muscle weakness, typically originating at the neuromuscular junction. This investigation is one of a series from the Engel and Sine groups at the Mayo Clinic that are unraveling the genetic basis of the disease. Around 15\% of myasthenia gravis disorders are not of the autoimmune type and are linked to mutations in genes encoding proteins in the neuromuscular junction. These can range from the enzymes, transporters, and docking proteins found presynaptically in the motor neuron that control synthesis and release of acetylcholine, to cholinesterases of postsynaptic origin that catalyze the rapid hydrolysis of the released acetylcholine neurotransmitter. Between the synthesis and release of acetylcholine and the destruction of the neurotransmitter lies the nicotinic receptor, which has the capacity to recognize acetylcholine and respond rapidly to neurotransmitter binding through channel gating. This initiates a depolarization and postsynaptic potential, 